\title{
The Relation between the Preferred Chewing Side and Occlusal Force Measured by T-Scan III System
}

\author{
Teuta Bicaj ${ }^{1}$, Teuta Pustina1, Enis Ahmedi' ${ }^{1}$, Linda Dula1, Zana Lila1, \\ Arlinda Tmava-Dragusha1, Resmije Ademi-Abdyli ${ }^{2}$, Nexhmije Ajeti ${ }^{3}$ \\ ${ }^{1}$ Department of Prosthetic Dentistry, Dental Branch, Medical Faculty, University of Prishtina, Prishtina, Kosovo \\ ${ }^{2}$ Department of Oral Surgery, Dental Branch, Medical Faculty, University of Prishtina, Prishtina, Kosovo \\ ${ }^{3}$ Department of Endodontic and Dental Pathology, Dental Branch, Medical Faculty, University of Prishtina, \\ Prishtina, Kosovo \\ Email: teutab68@yahoo.com
}

Received 6 March 2015; accepted 31 March 2015; published 2 April 2015

Copyright (C) 2015 by authors and Scientific Research Publishing Inc.

This work is licensed under the Creative Commons Attribution International License (CC BY). http://creativecommons.org/licenses/by/4.0/

cC) (7) Open Access

\begin{abstract}
Occlusal force has the main role in determining the teeth health in general and determining the chewing, muscle and TMJ function. The objective of this study was to find if there was any relation between the preferred chewing side and the force, measured by T-scan III system. The total number of 90 (46 female and 44 male) dental students was included in this study. They were asked whether their preferred chewing side was right, left or both. Afterwards, the occlusal forces in habitual occlusion, using T-Scan III Electronic device (IP-CO position mode, MA-mode, MA sensitivity setting) were measured. The data were presented as Mean \pm SD. Spearman correlation was used to find the correlation between the occlusal force and chewing side. Differences in $\mathrm{P}<0.05$ were considered significant. There were 90 subjects ( 46 female and 44 male) mean age 23.3 (SD \pm 2.2 years) included in the study. The preferred chewing side of 42 subjects was the right side, 24 of them preferred the left side and the other 24 preferred both sides as the chewing side. Occlusal force was higher in the preferred chewing side $(P<0.05)$ but using Spearman correlation, there was no statistical significance of occlusal force between the groups, the left side $(r=-0.143, P>0.05)$ and the right side $(r=0.143, P>0.05)$ according to the preferred chewing side. In this study, the relation between the preferred chewing side and occlusal force was found.
\end{abstract}

\section{Keywords}

T-Scan III, Occlusal Contact Force, Preferred Chewing Side

\footnotetext{
${ }^{*}$ Corresponding author.
}

How to cite this paper: Bicaj, T., Pustina, T., Ahmedi, E., Dula, L., Lila, Z., Tmava-Dragusha, A., Ademi-Abdyli, R. and Ajeti, N. (2015) The Relation between the Preferred Chewing Side and Occlusal Force Measured by T-Scan III System. Open Journal of Stomatology, 5, 95-101. http://dx.doi.org/10.4236/ojst.2015.54014 


\section{Introduction}

Maximum bite force is one of the indicators of the functional state of the masticator system [1]-[3]. The force results from the action of the jaw elevator muscles (in turn, determined by the central nervous system and feedback from muscle spindles, mechanoreceptors, and nociceptors) modified by the craniomandibular biomechanics [4]. It is considered that the bite force is the key determinant of the masticator function [5]. Masticator function is predicted by a number of parameters, including bite force [6] and occlusal contact area [7], which suggests that the higher the bite force is and the larger the occlusal contact area is, the more efficient the mastication is. Reduced masticator function is also related to smaller occlusal contact area [7]. Lower activity of the cervical muscles has been associated with a smaller occlusal contact area [8]. Maximum bite force is a useful indicator of the functional state of the masticator system and the loading of the teeth, and its recordings can be performed in a relatively simple way in the clinic.

Occlusal force has the main role in determining the teeth health in general and determining the chewing, muscle and TMJ function. Any problem that makes a bite unstable causes pain, broken restorations, gum disease, tooth loss, headaches and TMJ disorders. That is the reason why the dental occlusal analysis is very crucial and should not be left as a matter of guess-work, as it still is. Articulation paper, waxes, pressure indicator paste, etc. are not sensitive enough to detect simultaneous contact and none of them measures both biting time and force. T-Scan III meets these challenges.

In order to achieve good food manipulation and transport, tongue and other tissue movements must be facilitated during mandibular movements for repositioning of mandibular teeth during sequential chewing strokes [9], suggesting that wide, bilateral chewing cycles are related to better masticator performance [10].

Unilateral chewing is found to be present in $45 \%$ to $97 \%$ [6] [11]-[13] of the population and is associated with centrally controlled factors, such as handedness [11], unilateral signs of temporomandibular disorders, asymmetrical loss of antagonist contact, and presence of removable partial dentures [12]. In a dentate population, chewing side preference was present in almost half the subjects and was associated with lateral asymmetry of bite force and occlusal contact area [12]. However, no studies concerning either occlusal or functional asymmetry in individuals with different facial heights have been found in the literature.

T-Scan III (Computerized Occlusal Analysis System) is a reliable and easy to use clinical diagnostic device that senses and analyses occlusal contact forces using ultra thin reusable sensors, shaped to fit the dental arch. According to Kerstein [14], computerized occlusal analysis is capable of describing contact time and forces which conventional occlusal instrumentation, such as paper, wax and paste, cannot.

The objective of this study was to find if there was any relation between the preferred chewing side and the force measured by T-scan III system.

\section{Methods}

The participants of the study were all undergraduate students of the Dental Branch of the Medical Faculty from the University of Prishtina. Before the measurements, they have signed the consent paper. The research has been accepted and approved by the Institutional Ethic Committee, (University of Prishtina, Medical Faculty, School of Dentistry) (Document nr. 3669/2011).

The measurements were done by a single examiner to reduce inter-observer error. The main criterion for inclusion in the investigation was presence of permanent natural teeth. Other criteria were:

- Absence of abrasion of the occlusal surfaces of the teeth.

- Absence of the large teeth fillings that can have impact on the teeth anatomy.

- Angle I relationship between upper and lower jaw.

- No history of an orthodontic treatment.

- No history of functional pathology.

According to Forrester et al. [15] the healthy jaw function is considered when:

- There are no more than four extracted teeth.

- There is no sound clicking of TMJ during the normal function.

- No history of pain of the TMJ that would need attention.

After eliminating cases unsuitable for this study, 90 examinees were selected (46 female and 44 male), mean age $23.3(\mathrm{SD} \pm 2.1)$.

Preferred chewing side is determined by subjective declaration of the examinees. They were asked whether 
their preferred chewing side was right, left or both. The examinees that were not sure about their preferred chewing side were classified on the group of "both". In other side, there are papers that describe detecting of the chewing side preference, by viewing of the examinees during mastication or using kinesiographic movements by using jaw tracking movement [5] [12].

The position of the examinee was sitting in a dental chair with the head position, normal to the body and the mandible, parallel to the floor (Figure 1).

Afterwards, the occlusal forces in habitual (centric) occlusion, using T-Scan III Electronic device (IP-CO position mode, MA-mode, MA sensitivity setting) were measured. (TScan $\left({ }^{\circledR}\right)$ III from Tekscan Inc., South Boston, MA, USA) (Figure 2).

For recordings, MA (Max Area frame) mode was used as well as "MA Sensitivity" setting and the default recording time for MA, of 10 seconds (1000 frames). Data collected were classified, analyzing occlusal force for the left and right half of the arch (Figure 3) as well as for the quadrants: anterior right, anterior left, posterior right and posterior left (Figure 4).

Occlusal force, presented by percentage (automatically by the T-scan electronic system) was analyzed in relation to a preferred chewing side. The T-scan sheets (sensor) used, have a layer thickness of $100 \mu \mathrm{m}$ and are therefore within the range of commercially available articulating foils, papers and silk ( $8-200 \mu \mathrm{m})$.

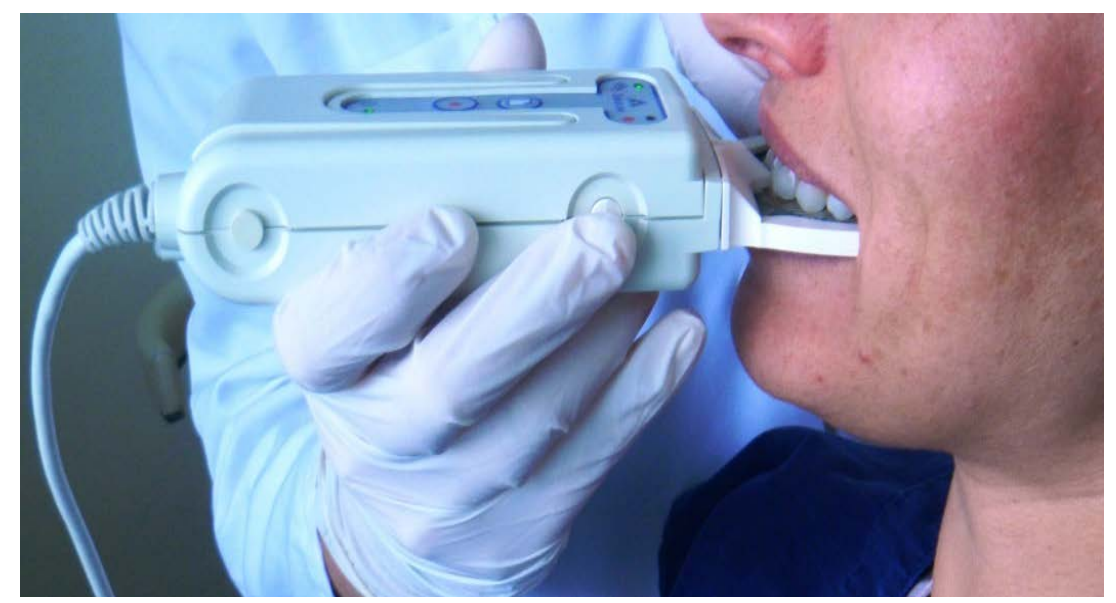

Figure 1. The position of examinee during recording with the T-scan III device. (We obtained written consent for publication of their image from person in the picture).

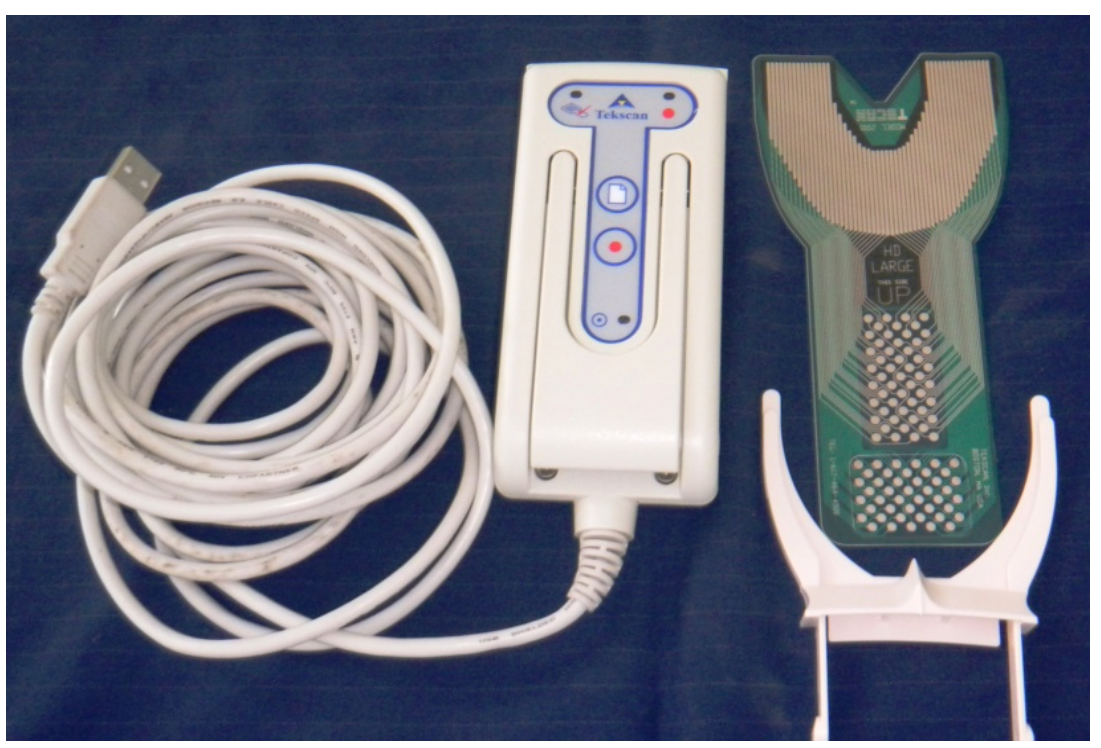

Figure 2. Tscan $\left({ }^{\circledR}\right)$ III from Tekscan Inc., South Boston, MA, USA. 


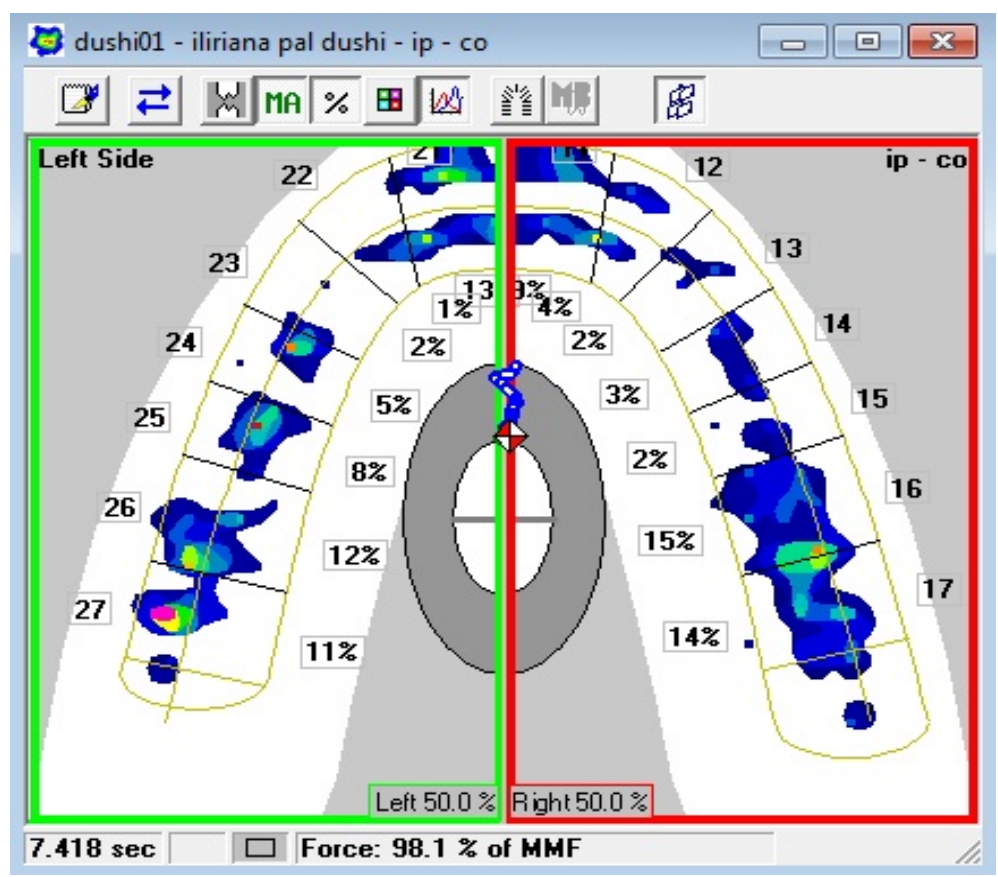

Figure 3. 2D images of recordings with the T-scan III device. Distribution of forces on the left and the right half.

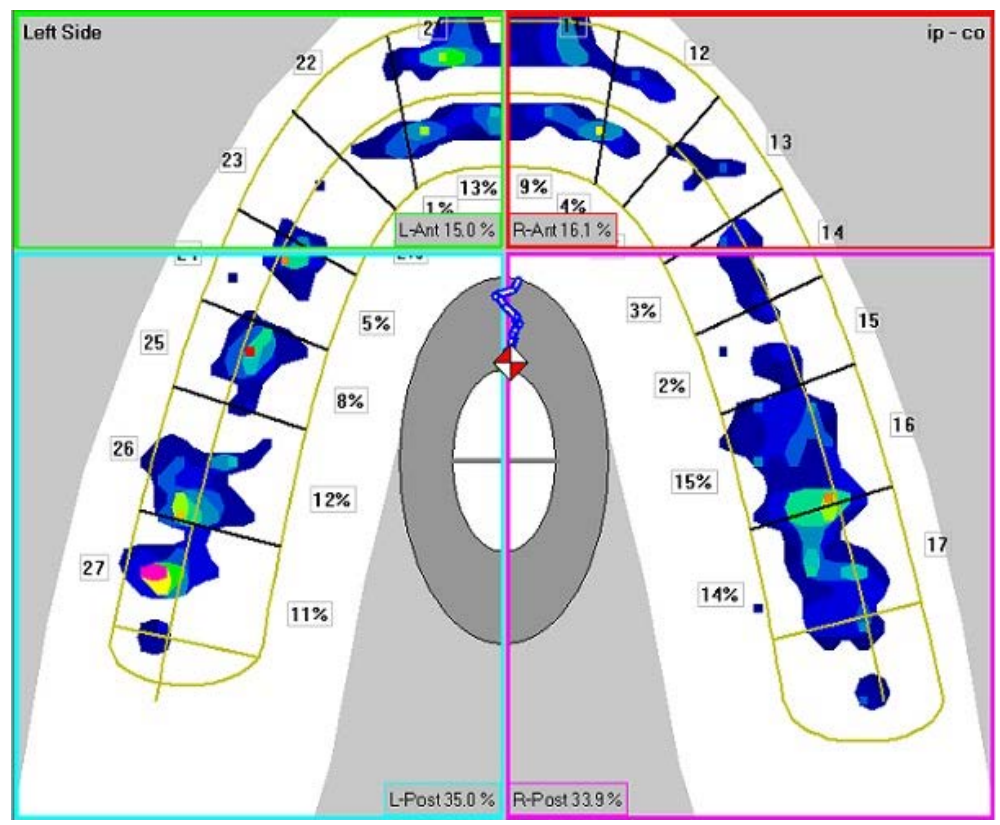

Figure 4. 2D images of recordings with the T-scan III device. Distribution of forces on the quadrants: anterior left and right and posterior left and right.

\section{Data Analysis}

Statistical analysis was made using Statistical Package for Social Science (SPSS) 19 for Windows (SPSS Inc., Chicago, Illinoiss, USA) and MS Excel (Microsoft Office, Windows 2007, USA). The data are presented as Mean \pm SD. Spearman correlation was used to find the relation between the occlusal force and chewing side (as not normally distributed numerical variables). Differences in $\mathrm{P}<0.05$ were considered significant (according to Dunn's Multiple Comparison test). 
Data are analyzed with descriptive statistics, and differences between groups are assessed with One Way ANOVA for normally distributed numerical variables, or nonparametric test-Kruskal Wallis test in case of not normally distributed numerical variables. Normality is tested with Kolmogorov-Smirnov test. Dunn's Multiple Comparison test was used as a post hoc test.

\section{Results}

A total number of 90 voluntaries (46 female and 44 male), mean age 23.3 ( $S D \pm 2.1$ ), were included in this study.

The study results are presented in Table 1 and Table 2. Occlusal force was higher in the preferred chewing side $(\mathrm{P}<0.05)$. There was no statistical significance of occlusal force according to the quadrants: anterior left, anterior right, posterior left and posterior right $(\mathrm{P}>0.05)$ (Table 1).

Using Spearman correlation, no statistical significance of occlusal force on the left side $(r=-0.143, P>0.05)$ and the right side $(r=0.143, P>0.05)$ according to the preferred chewing side (between the groups), was found. Analyzing the quadrants of the dental arch, there was also no statistical significance of occlusal force, left anterior $(r=-0.03, P>0.05)$, right anterior $(r=0.109, P=0.302)$, left posterior $(r=-0.06, P=0.562)$ and right posterior $(\mathrm{r}=-0.01, \mathrm{P}=0.858)$ according to the preferred chewing side (Table 2).

\section{Discussion}

No studies relating to the preferred chewing side to occlusal force, measured by T-scan, have been found in the literature. The present study evaluated this relationship by analyzing occlusal force relating to the preferred chewing side of examinees. It was found a weak difference between the preferred chewing side and occlusal forces but with no statistical significance.

Different occlusal indicators can be used during prosthodontic treatment for diagnosis and treatment purposes [16]. Qualitative indicators (articulating paper and articulating silk) can measure only the location and number of tooth contacts while quantitative indicators (electro-optic and resistive techniques such as T-scan) can measure the time and force characteristics of tooth/teeth contact [17]-[19].

In this study the preferred chewing side was detected by declaring of the examinees, while in the literature we found that it can be detected also by observing the examinees during mastication [5] [12] or using kinesiographic movements by using jaw tracking movement. We could not find any evidence to proof the impact of our choice, so detecting of the preferred chewing side diversely, can also be an aim for additional research.

Table 1. Occlusal force according to the preferred chewing side.

\begin{tabular}{cccccccc}
\hline \multirow{2}{*}{$\begin{array}{c}\text { Preferred } \\
\text { chewing side }\end{array}$} & $\mathbf{N}$ & \multicolumn{5}{c}{ Occlusal force (\%) Mean \pm SD } \\
\cline { 3 - 8 } & & Left & Right & Anterior left & Anterior right & Posterior left & Posterior right \\
\hline Right & 42 & $49.4 \pm 7.3$ & $\mathbf{5 0 . 8} \pm \mathbf{7 . 6}$ & $13.8 \pm 9.5$ & $13.9 \pm 10.2$ & $35.4 \pm 12.3$ & $36.9 \pm 11.4$ \\
Left & 24 & $\mathbf{5 5 . 4} \pm \mathbf{9 . 0}$ & $44.7 \pm 9.3$ & $15.2 \pm 9.4$ & $13.0 \pm 7.9$ & $40.1 \pm 12.1$ & $31.7 \pm 10.9$ \\
Both sides & 24 & $52.0 \pm 7.9$ & $47.2 \pm 10.1$ & $14.8 \pm 10.9$ & $15.9 \pm 9.7$ & $38.0 \pm 14.4$ & $31.3 \pm 11.1$ \\
Total & $\mathbf{9 0}$ & $\mathbf{5 1 . 4} \pm \mathbf{8 . 0}$ & $\mathbf{4 8 . 2} \pm \mathbf{9 . 1}$ & $14.5 \pm 9.8$ & $14.2 \pm 9.5$ & $37.3 \pm 12.9$ & $34.0 \pm 11.4$ \\
P-value & & $\mathbf{P = \mathbf { 0 . 0 2 9 } *}$ & $\mathbf{P}=\mathbf{0 . 0 2 8} *$ & $\mathrm{P}=0.851$ & $\mathrm{P}=0.417$ & $\mathrm{P}=0.342$ & $\mathrm{P}=0.096$ \\
\hline
\end{tabular}

$* \mathrm{P}<0.05$; *Using Dunn's Multiple Comparison test, it was found a statistical significance between the bite force and preferred chewing side (for the left and the right preferred chewing side).

Table 2. Correlation between occlusal force and preferred chewing side.

\begin{tabular}{ccccccc}
\hline & \multicolumn{5}{c}{ Occlusal force (\%) } \\
\cline { 2 - 7 } & Left & Right & Anterior left & Anterior right & Posterior left & Posterior right \\
\hline \multirow{2}{*}{ Chewing side } & $\mathrm{r}=-0.143$ & $\mathrm{r}=0.143$ & $\mathrm{r}=-0.03$ & $\mathrm{r}=0.109$ & $\mathrm{r}=-0.06$ & $\mathrm{r}=-0.01$ \\
& $\mathrm{P}=0.176$ & $\mathrm{P}=0.176$ & $\mathrm{P}=0.771$ & $\mathrm{P}=0.302$ & $\mathrm{P}=0.562$ & $\mathrm{P}=0.858$ \\
\hline
\end{tabular}


Palinkas et al. [20] have investigated the influence of gender and age on maximal bite force and masticator muscles thickness.

We think that higher occlusal force in preferred chewing side should be associated with expressing of the higher muscular force on that side. As mentioned in the "background" part of the manuscript, "Lower activity of the cervical muscles has been associated with a smaller occlusal contact area [8]." So, the hypothesis was that, the lower force applied in a non preferred chewing side is associated with a "weaker" muscles on that side therefore, with a smaller occlusal contact area; And the smaller occlusal contact area is associated with a reduced occlusal force. For this reason, additional clinical investigations can be done.

\section{Conclusion}

In this study, the relation between the bite force and preferred chewing side was found. Occlusal force was higher in the preferred chewing side $(\mathrm{P}<0.05)$. There was no statistical significance of occlusal force according to the quadrants: anterior left, anterior right, posterior left and posterior right $(\mathrm{P}>0.05)$.

\section{Disclosure and Acknowledgements}

The main source of funding for the study was the University of Prishtina by providing the T-scan III device.

Language corrections were performed with the support of Prof. Sadete Pllana (university professor of English and German language in the University of Prishtina).

\section{Authors' Contribution}

TB-carried out of acquisition of data and made substantial contributions to conception and design, analysis and interpretation of data.

$\mathrm{TP}$ - participated on conception of the study and gave final approval of the version to be published.

EA-had been involved in drafting the manuscript and revising it critically for important intellectual content.

LD - participated on acquisition of data and made contributions to conception and design.

ZL-participated on acquisition of data and made contributions to conception and design.

ATD - participated on acquisition of data and made contributions to conception and design.

RA-participated on conception of the study and gave final approval of the version to be published.

NA - participated on acquisition of data and made contributions to conception and design.

\section{Competing Interests}

The authors confirm that there are no conflicts of interest associated with the submission of this article.

\section{References}

[1] Julien, K.C., Buschang, P.H., Throckmorton, G.S. and Dechow, P.C. (1996) Normal Masticatory Performance in Young Adults and Children. Archives of Oral Biology, 41, 69-75. http://dx.doi.org/10.1016/0003-9969(95)00098-4

[2] Hatch, J.P., Shinkai, R.S., Sakai, S., Rugh, J.D. and Paunovich, E.D. (2001) Determinants of Masticatory Performance in Dentate Adults. Archives of Oral Biology, 46, 641-648. http://dx.doi.org/10.1016/S0003-9969(01)00023-1

[3] Okiyama, S., Ikebe, K. and Nokubi, T. (2003) Association between Masticatory Performance and Maximal Occlusal Force in Young Men. Journal of Oral Rehabilitation, 30, 278-282. http://dx.doi.org/10.1046/j.1365-2842.2003.01009.x

[4] Okeson, J.P. (2008) Temporomandibularni poremecaji i okluzija. 5th Edition, 1st Croatic Edition, Medicinska Naklada Zagreb, Chapter 2, 29-45.

[5] Gomes, S.G., Custodio, W., Faot, F., Cury, A.A. and Garcia, R.C. (2011) Chewing Side, Bite Force Symmetry, and Occlusal Contact Area of Subjects with different Facial Vertical Patterns. The Brazilian Oral Research, 25, 446-452. http://dx.doi.org/10.1590/S1806-83242011005000014

[6] Diernberger, S., Bernhardt, O., Schwahn, C. and Kordass, B. (2008) Selfreported Chewing Side Preference and Its Associations with Occlusal, Temporomandibular and Prosthodontic Factors: Results from the Population-Based Study of Health in Pomerania (SHIP-0). Journal of Oral Rehabilitation, 35, 613-620.

http://dx.doi.org/10.1111/j.1365-2842.2007.01790.x

[7] Lujan-Climent, M., Martinez-Gomis, J., Palau, S., Ayuso-Montero, R., Salsench, J. and Peraire, M. (2008) Influence of Static and Dynamic Occlusal Characteristics and Muscle Force on Masticatory Performance in Dentate Adults. Euro- 
pean Journal of Oral Sciences, 116, 229-236. http://dx.doi.org/10.1111/j.1600-0722.2008.00530.x

[8] So, K., Komiyama, O., Arai, M., Kawara, M. and Kobayashi, K. (2004) Influence of Occlusal Contact on Cervical Muscle Activity during Submaximal Clenching. Journal of Oral Rehabilitation, 31, 417-422. http://dx.doi.org/10.1111/j.1365-2842.2004.01265.x

[9] Mioche, L., Hiiemae, K.M. and Palmer, J.B. (2002) A Postero-Anterior Videofluorographic Study of the Intra-Oral Management of Food In Man. Archives of Oral Biology, 47, 267-280. http://dx.doi.org/10.1016/S0003-9969(02)00007-9

[10] Wilding, R.J. and Lewin, A. (1994) The Determination of Optimal Human Jaw Movements Based on Their Association with Chewing Performance. Archives of Oral Biology, 39, 333-343. http://dx.doi.org/10.1016/0003-9969(94)90125-2

[11] Nissan, J., Gross, M.D., Shifman, A., Tzadok, L. and Assif, D. (2004) Chewing Side Preference as a Type of Hemispheric Laterality. Journal of Oral Rehabilitation, 31, 412-416. http://dx.doi.org/10.1111/j.1365-2842.2004.01256.x

[12] Martinez-Gomis, J., Lujan-Climent, M., Palau, S., Bizar, J., Salsench, J. and Peraire, M. (2009) Relationship between Chewing Side Preference and Handedness and Lateral Asymmetry of Peripheral Factors. Archives of Oral Biology, 54, 101-107. http://dx.doi.org/10.1016/j.archoralbio.2008.09.006

[13] Felctio, C.M., Melchior, M.O., Silva, M.A.M.R. and Celeghini, R.M.S. (2007) Masticatory Performance in Adults Related to Temporomandibular Disorder and Dental Occlusion (Original Title: Desempenho mastigatório em adultos relacionado com a desordem temporomandibular e com a oclusão). Pró-Fono Revista de Atualização Científica, 19, 151158.

[14] Kerstein, R. (2001) Current Applications of Computerized Occlusal Analysis in Dental Medicine. General Dentistry, 49, 521-530.

[15] Forrester, S.E., Allen, S.J., Presswood, R.G., Toy, A.C. and Pain, M.T.G. (2010) Neuromuscular Function in Healthy Occlusion. Journal of Oral Rehabilitation, 37, 663-669. http://dx.doi.org/10.1111/j.1365-2842.2010.02097.x

[16] Ando, K., Fuwa, Y., Kurasawa, M., Kondo, T. and Goto, S. (2009) Bite Force Measurement System Using PressureSensitive Sheet and Silicone Impression Material. Dental Materials Journal, 28, 212-218. http://dx.doi.org/10.4012/dmj.28.212

[17] Babu, R.R. and Nayar, S.V. (2007) Occlusion Indicators: A Review. Journal of Indian Prosthodontic Society, 7, 170174. http://dx.doi.org/10.4103/0972-4052.41066

[18] Koos, B., Godt, A., Schille, C. and Göz, G. (2010) Precision of an Instrumentation-Based Method of Analyzing Occlusion and Its Resulting Distribution of Forces in the Dental Arch. Journal of Orofacial Orthopedics, 71, 403-410. http://dx.doi.org/10.1007/s00056-010-1023-7

[19] Koos, B., Höller, J., Schille, C. and Godt, A. (2012) Time-Dependent Analysis and Representation of Force Distribution and Occlusion Contact in the Masticatory Cycle. Journal of Orofacial Orthopedics, 73, 204-214. http://dx.doi.org/10.1007/s00056-012-0075-2

[20] Palinkas, M., Nassar, M.S., Cecílio, F.A., Siéssere, S., Semprini, M., Machado-de-Sousa, J.P., Hallak, J.E. and Regallo, S.C. (2010) Age and Gender Influence on Maximal Bite Force and Masticatory Muscles Thickness. Archives of Oral Biology, 55, 797-802. http://dx.doi.org/10.1016/j.archoralbio.2010.06.016

\section{List of Abbreviations}

TMJ-Temporomandibular Joint.

MA-Max Area Frame.

IP-CO_-Intercuspal Position, Centric Occlusion. 